\title{
An evaluation of current methods for the diagnostic histochemistry of amyloid
}

\author{
JOHN H. COOPER \\ From the Department of Pathology, Pathology Institute, and Dalhousie University, Halifax, \\ Nova Scotia, Canada
}

SYNOPSIS Six current histological methods for demonstrating amyloid (crystal violet, thioflavine- $\mathrm{T}^{\frac{8}{8}}$ fluorescence, Congo-red staining and fluorescence, Sirius-red staining, and Congo- or Sirius-red $N$ birefringence) were applied in 25 cases of amyloidosis of various types and 47 pseudo-amyloid lesions. The results were compared and were correlated with those of ancillary histochemical tests $\vec{O}$ and clinico-pathological data and each method's sensitivity and specificity for amyloid was evalu- 은 ated. Thioflavine-T and, to a lesser degree, Congo-red fluorescence and Sirius-red staining proved $\vec{c}$ very sensitive but not specific. Green birefringence with Congo or Sirius red was specific but not completely sensitive. The coexistence of Congo-red (and Sirius-red) staining and a positive DMAB-nitrite reaction occurred in all amyloid specimens and appeared specific for amyloid.

Three main techniques are currently employed for the histological demonstration of amyloid: 'metachromasia' with triphenylmethane dyes (eg, crystal violet); staining with substantive cotton dyes (Congo red, Sirius red), with or without fluorescence or polarization microscopy; and fluorescence with thiazole dyes (eg, thioflavine-T). The pseudometachromatic (Kramer and Windrum, 1955) triphenylmethane methods appear to have lost favour, and the recent literature is concerned mainly with the respective merits and defects of the cotton dyes and fluorescent thiazoles. Some authors recommend refinements of Congo-red (Missmahl, 1957; Puchtler and Sweat, 1965; Puchtler, Sweat, and Levine, 1962) or Sirius-red (Sweat and Puchtler, 1965) techniques; others express strong preference for the thioflavines (Vassar and Culling, 1959; Schwartz, 1965; Nebut and Hartmann, 1966; Saeed and Fine, 1967); still others criticize thioflavine methods on grounds of non-specificity (Rogers, 1965; McKinney and Grubb, 1965).

The present evaluation of representative methods was planned to help to resolve this controversy. Its design recognizes two points of difficulty that tend to obscure the issue and to frustrate judgment: these points are that the microscopical detection of amyloid is a biphasic operation, and that evaluation of relevant methods requires an indirect, heuristic approach.

Received for publication 5 November 1968.
Microscopical discovery of amyloid deposits comprises two distinct, sequential operations, $\frac{\circ}{\Phi}$ screening and identification. The former, though it can be performed suboptimally on sections stained $\overline{\mathrm{O}}$ by routine methods, ideally requires a method with a high degree of sensitivity, both qualitative, whereby all varieties of amyloid are demonstrated, and quantitative, whereby even minute deposits are revealed $\widehat{0}$ by the intense visual effect. Identification, on the $:-$ other hand, absolutely requires a method of high 3 specificity, whereby only amyloid, and no other $\delta$ substance, reacts positively. Sensitivity and specificity are recognized by organic chemists (Feigl, 1966) as 0 reciprocal rather than parallel properties, in that a highly sensitive method tends to be rather non-o specific and a highly specific method relatively insensitive; therefore, any method of evaluation must o measure each of these properties separately.

Direct evaluation of an unknown requires an absolute standard with which the unknown can be directly compared and thereby calibrated. $\mathrm{No}_{0}$ absolute standard is available for an evaluation of $\frac{\text { C }}{\mathscr{D}}$ methods of diagnosing amyloid, because there is $\stackrel{\oplus}{-}$ no standard method of proved sensitivity and 0 specificity for amyloid to serve as an absolute basiso of comparison. The requisite procedure therefore $\stackrel{\mathbb{Q}}{\Omega}$ entails preliminary recording of staining patterns $\mathbb{\Phi}$ produced by each method on a set of tissues; $\frac{\varrho}{0}$ subsequent correlation of these patterns with all relevant histochemical, pathological, and clinicalo 
information; and, finally, synthesis of the data to determine the sensitivity and specificity of each method.

\section{MATERIALS AND METHODS}

Most of the tissues were collected prospectively from the surgical and necropsy diagnostic services in cases in which amyloid disease was suspected. A few were deliberately selected retrospectively to complete the series. In all, 25 cases of amyloidosis of various types (Table I) and 47 of various pseudo-amyloid lesions (Table II) were examined. The tissues were fixed in neutralized formalin for six to 36 hours and were processed to paraffin. In most cases only one or two blocks of one organ or biopsy specimen were examined, but multiple blocks from various organs were studied in some necropsy cases of amyloidosis. Every block was serially sectioned to permit performance of eight constant methods and, as required, ancillary methods to characterize pseudoamyloid deposits.

TABLE I

TYPE OF AMYLOID LESION (25 CASES)

$\begin{array}{ll}\text { Myelomatous } & 2 \\ \text { Primary generalized } & 6 \\ \text { Secondary generalized } & 6 \\ \text { Cutaneous } & 2 \\ \text { Senile cardiac } & 4 \\ \text { Pancreatic insular } & 3 \\ \text { Cerebral } & 2\end{array}$

TABLE II

PSEUDO-AMYLOID LESIONS (57 CASES)

Strong Hyaline (24)

Scar tissue

Solar elastosis

Endocardial elastosis

Scleroderma

Atrophic test is

Insulinoma

Breast carcinoma

Turban tumour
Fibrinoid (23)

Splenic arteriosclerosis

Diabetic nephropathy

Allergic vasculitis

'Schwartzmann kidney'

Radionecrosis

Rheumatoid nodule

Splenic follicular 'hyaline'

Laryngeal node
CONSTANT METHODS Haematoxylin and eosin; van Gieson; crystal-violet metachromasia; thioflavine-T fluorescence (Vassar and Culling, 1959); Congo-red staining (Puchtler et al, 1962) and fluorescence (Puchtler and Sweat, 1965); Sirius-red staining (Sweat and Puchtler, 1965); DMAB-nitrite (Adams, 1957).

ANCILlaRY METHODS Periodic-acid Schiff; phosphototungstic acid-haematoxylin; acid orcein; ferric ferricyanide reduction (Lillie, 1965); diazotization-S acid (Glenner and Lillie, 1959); dihydroxydinaphthyldisulphide-fast blue (DDD), and thioglycolate DDD (Barrnett and Seligman, 1952); alcian blue 8GS (Barka and Anderson, 1963); performic acid-alcian blue (Pearse, 1960).

To allow for differing $p \mathrm{H}$ optima of the reaction, crystal-violet staining was performed in duplicate with dilute $(0.1 \%)$ aqueous solutions buffered to $p \mathrm{H} 1.5$ and

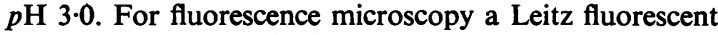
microscope was used with the appropriate filters prescribed by Vassar and Culling (1959) for thioflavine-T and by Puchtler and Sweat (1965) for Congo red. Polarization-optical studies were performed with an ordinary Zeiss microscope equipped with polaroid filters and with a Leitz polarizing microscope. These studies were directed particularly to appreciation of the enhanced green birefringence, which has been described as a specific reaction of amyloid stained with Congo red (Missmahl, 1957) or Sirius red (Sweat and Puchtler, 1965). The DMAB-nitrite reaction (for indole residues) was applied in all cases, because amyloid appears always to have an appreciable tryptophan content (Cohen, 1965) and, therefore, should always give a positive indole reaction.

The methods were evaluated by preliminary recording of all stained deposits morphologically consistent with amyloid, and subsequent analysis of these deposits to determine whether they were true (amyloid) or false (pseudo-amyloid). Deposits were designated 'pseudoamyloid' when analysis indicated that they consisted entirely of identifiable substances known to be distinct from amyloid (Table III). Alternatively, deposits that stained with one or more amyloid methods and that could not be otherwise identified were assumed to be amyloid. Specificity was estimated from the observed incidence of falsely positive results yielded by each method. Qualitative sensitivity was calculated as a ratio

TABLE III

HISTOCHEMICAL PROFILES OF PSEUDO-AMYLOID COMPONENTS

\begin{tabular}{|c|c|c|c|}
\hline Staining Method & Collagen & Elastica & Fibrin \\
\hline $\begin{array}{l}\text { Van Gieson } \\
\text { Orcein } \\
\text { PAS } \\
\text { Ferric ferricyanide } \\
\text { Diazotization-S acid } \\
\text { DMAB-nitrite } \\
\text { DDD } \\
\text { Thioglycolate-DDD } \\
\text { Performic acid-alcian blue } \\
\text { Alcian blue } \\
\text { Birefringence } \\
\text { Autofluorescence }\end{array}$ & $\begin{array}{l}++ \\
\mp \\
\mp \\
\frac{\mp}{\mp} \\
- \\
- \\
+ \\
+ \\
++ \\
\mp \text { (grey) }\end{array}$ & $\begin{array}{l}\overline{+} \\
\overline{+} \\
+ \\
++ \\
\bar{\mp} \\
+ \\
\overline{-} \\
\overline{+} \\
++ \text { (blue-white) }\end{array}$ & $\begin{array}{l}- \\
- \\
++ \\
++ \\
+ \\
++ \\
\overline{+} \\
+ \\
- \\
+ \text { or } \mp \\
+ \text { (white) }\end{array}$ \\
\hline
\end{tabular}




\begin{tabular}{|c|c|c|c|}
\hline STAINING METHODS & HYALINES & AMYLOIDS & FIBRINOIDS \\
\hline Crystal Violet & & & \\
\hline Thioflavine T. Fluorescence & & & \\
\hline Congo Red or Sirius Red & & & \\
\hline $\begin{array}{c}\text { Green Birefringence with } \\
\text { Congo Red or Sirius Red }\end{array}$ & & \\
\hline $\begin{array}{c}\text { Congo Red or Sirius Red } \\
\text { Dmab - Nitrite }\end{array}$ & & \\
\hline $\begin{array}{l}\text { Negative } \\
\text { Yellow }\end{array}$ & Q & \\
\hline
\end{tabular}

FIG. 1. The specificity and qualitative sensitivity of methods for staining amyloid. A perfect method would yield a band completely spanning the central column $(100 \%$ sensitivity) without overlapping into either lateral column $(100 \%$ specificity).

(number of amyloid cases detected by the method/total number of amyloid cases surveyed). Quantitative sensitivity was roughly assessed from the relative visual effect produced by each method.

\section{RESULTS AND DISCUSSION}

Two main classes of pseudo-amyloid were recognized and were segregated according to their histochemical profiles (Table II). They were designated 'stromal hyaline', formed by alteration of normal connective-tissue components, ie, collagen and/or elastica, and 'fibrinoid', formed by infiltration of plasmatic protein, including fibrin, into the interstitium.

The results of evaluation are depicted in Fig. 1 as a measurement of two parameters: range of qualitative sensitivity, ie, proportion of total amyloid cases detected by the method, which is indicated by the degree of spanning of the central 'amyloid' column; and the amount of non-specificity, $i e$, proportion of falsely positive results yielded by the method, which is indicated by the degree of overlap into the lateral 'pseudo-amyloid' columns.

Crystal-violet 'metachromasia' was specific within the limits of the study, ie, excluding epithelial and chondroid mucins, on morphological grounds, but had a low range $(<70 \%)$ of sensitivity. This defect is due largely to the almost uniform non-reactivity of deposits of primary generalized amyloidosis. Therefore, the crystal-violet method by itself cannot be recommended.

The pattern for thioflavine-T was quite different. Sensitivity was $100 \%$, all amyloid lesions being stained, in many cases with a brilliant intensity surpassing that of any other method. Unfortunately, an appreciable proportion of the amyloid lesions did not yield intense yellow or green fluorescence $\frac{0}{5}$ but only a silvery-blue of variable intensity; this cannot always be distinguished from the similar $\overrightarrow{\vec{F}}$ silvery-blue fluorescence exhibited by some stromal hyaline deposits and, more importantly, by many fibrinoids, which, morphologically, more closely simulate amyloid. The thioflavine-T method, there- $\overparen{\Phi}$ fore, though attractively simple and highly sensitive, is not specific and requires confirmation of its $\mathrm{s}$ positive results by a truly specific method. Recent $\vec{O}$ work by Kelényi (1967) and Burns, Pennock, and $\overrightarrow{\vec{\omega}}$ Stoward (1967) on the histochemistry of the thio- $\vec{G}$ flavines fortifies this conclusion.

The improved Congo-red technique of Puchtler et al (1962), and the analogous Sirius-red method by ? the same group (Sweat and Puchtler, 1965), produced essentially the same results except that $\vec{\circ}$ staining with the Sirius dye was more intensely red. Congo red, however, unlike Sirius red, is a fluorochrome (Puchtler and Sweat, 1965) and yields a $\bar{c}$ much more intense staining effect by fluorescence $\stackrel{一}{\gtrless}$ microscopy, thereby rivalling thioflavine-T as a $\vec{\varphi}$ screening technique. Apart from these variations in 8 staining intensity the performance of the three techniques was essentially the same: all amyloids were stained but staining of elastica and elastotic material was not always specific. Therefore, these methods are not completely specific.

The green birefringence test with Congo red or $\cong$ Sirius red was completely specific for amyloid $\overrightarrow{0}$ (vegetable-cellulose fibres can be excluded morpho- $\exists$ logically), and must be regarded as the essential method for its certain identification. Even theoretically, however, the sensitivity of this method for $\overline{7}$ minute amounts of amyloid would be suspect, because the colour birefringence phenomenon is a 3 . function of both the thickness and the orientation of the deposits (Wolman and Bubis, 1965). In practice, sensitivity is further diminished by imper- 0 fections of the optical apparatus and of its use, so that probably it cannot exceed the $96 \%$ sensitivity achieved in this study. Thus, exclusive dependence on Congo-red birefringence would yield a small $\tilde{\sigma}$

proportion of falsely negative results.
The DMAB-nitrite reaction is, of course, not $\tilde{\omega}_{\mathrm{W}}$ specific for amyloid, but in this study it was con- $\frac{\omega}{\sigma}$ sistently sensitive for it; furthermore, this reaction was negative for all pseudo-amyloids except the $\stackrel{0}{\frac{D}{D}}$ 'fibrinoids', which did not stain with Congo red or $\stackrel{\$}{?}$ Sirius red. The DMAB-nitrite reaction, therefore, 0 provides a useful supplementary and confirmatory $\overrightarrow{0}$ test, since the results indicate that a substance which $\mathbb{D}$ stains both with Congo red or Sirius red and with $\frac{}{1}$ DMAB-nitrite is amyloid. (It should be borne in mind that overfixation in formalin (Adams, 1957) blocks the DMAB-nitrite reaction.) 
In summary, the evaluation yielded two conclusions.

1 Thioflavine-T fluorescence, Congo-red fluorescence, and Sirius-red staining (in descending order of quantitative sensitivity) constitute efficient screening methods for amyloid but lack the specificity necessary for positive identification.

2 Green birefringence with Congo red or Sirius red affords specific identification of amyloid but may be inconspicuous or (rarely) absent in minute deposits. In such cases, the coexistence of Congo-red (or Sirius-red) staining and a positive DMABnitrite reaction can be taken as proof of amyloid.

In the author's experience the screening tests can be performed readily by a competent orthodox histopathologist, whereas the identification procedures present difficulty to anyone not versed in the special techniques of polarization microscopy and histochemistry.

This work was supported in part by grant MA-1920 from the Medical Research Council of Canada. The author is indebted to Mr J. S. Gibson for his invaluable technical assistance.
REFERENCES

Adams, C. W. M. (1957). J. clin. Path., 10, 56.

Barka, T., and Anderson, P. J. (1963). Histochemistry: Theory, Practice, and Bibliography, p. 78. Harper and Row, New York.

Barrnett, R. J., and Seligman, A. M (1952). J. nat. Cancer Inst., $13,215$.

Burns, J., Pennock, C. A., and Stoward, P. J. (1967). J. Path. Bact., 94, 337.

Cohen, A. S. (1965). Int. Rev. exp. Path., 4, 159.

Feigl, F. (1966). Spot Tests in Organic Analysis, 7th ed. p. 43. Elsevier, Amsterdam and New York.

Glenner, G. G., and Lillie, R. D. (1959). J. Histochem. Cytochem., $7,416$.

Kelényi, G. (1967). Ibid., 15, 172.

Kramer, H., and Windrum, G. M. (1955). Ibid., 3, 227.

Lillie, R. D. (1965). Histopathologic Technic and Practical Histochemistry, 3rd ed., p. 211. McGraw-Hill (Blakiston), New York.

McKinney, B., and Grubb, C. (1965). Nature (Lond.), 205, 1023.

Missmahl, H. P. (1957). Z. wiss. Mikr., 63, 133.

Nebut, M., and Hartmann, L. (1966). Ann. Biol. clin., 24, 703.

Pearse, A. G. E. (1960). Histochemistry: Theoretical and Applied, 2nd ed., p. 820. Churchill, London.

Puchtler, H., and Sweat, F. (1965). J. Histochem. Cytochem., 13, 693.

- and Levine, M. (1962). Ibid., 10, 355.

Rogers, D. R. (1965). Amer. J. clin. Path., 44, 59.

Saeed, S. M., and Fine, G. (1967). Ibid., 47, 588.

Schwartz, P. (1965). Trans. N.Y. Acad. Sci., 27, 393.

Sweat, F., and Puchtler, H. (1965). Arch. Path., 80, 613.

Vassar, P. S., and Culling, C. F. A. (1959). Ibid., 68, 487.

Wolman, M., and Bubis, J. J. (1965). Histochemie, 4, 351. 